\title{
Explicit Guidance of Ballistic Entry Using Improved Matched Asymptotic Expansions
}

\author{
By Zeal-Sain KuO, Kuo-Chung LIU and Yun-Sheng CHANG \\ Chung Cheng Institute of Technology, National Defense University, Tao-Yuan, Taiwan, R.O.C.
}

(Received June 12th, 2006)

\begin{abstract}
This study presents a means of explicit guidance for ballistic entry using an improved method of matched asymptotic expansions. The trajectory of ballistic entry into a planetary atmosphere is still an important and often critical phase of a mission. In the paper, feedback control via drag modulation is used to guide the vehicle during the atmospheric entry, whereas a matched asymptotic solution for the entry trajectory is available to aim the target. The feedback control ensures the stability of a trajectory around the nominal trajectory by compensating for the non-linear terms in the motion of the vehicle. Using the improved method of matched asymptotic expansions, the control algorithms for the guidance law are derived explicitly and tested against the 1976 U.S. Standard Atmosphere. Simulation results indicate that the control algorithms can effectively control the trajectories in the lower atmosphere under the targeting dispersions of atmospheric variations.
\end{abstract}

Key Words: Explicit Guidance, Ballistic Entry, Matched Asymptotic Expansions, Tactical Ballistic Missile

\section{Introduction}

There is always a need to provide precise, real-time, lowcost guidance systems for high-performance ballistic missiles to reduce the miss distance of targeting error. ${ }^{1)}$ The purpose of this paper is to obtain the explicit guidance of ballistic missiles using an improved method of matched asymptotic expansions (iMAE) ${ }^{2,3)}$ The research of guidance for ballistic entry has received considerable interest. ${ }^{4-9)}$ The trajectory of tactical ballistic missiles (TBM), as shown in Fig. 1, consists of three phases: an ascending phase entering outerspace or near the vacuum with powered lift-off, followed by a free flight in an elliptic orbit, and then completed by a descending flight targeting the desired area. Feedback control is used to guide the vehicle during atmospheric reentry, while an iMAE solution for the entry trajectory is available to aim the target area. The approach presented here uses a feedback control technique to ensure the stability of a trajectory about the nominal trajectory by compensating the non-linear terms in the motion of the vehicle. Nominal trajectories generated by iMAE are used in developing the control expressions. ${ }^{10)}$ As the reference trajectory data is produced analytically, it decreases the need for numerical integration or trajectory data storage and therefore reduces the computational burden. The usefulness of an analytic solution depends on its accuracy. Since the solutions obtained by iMAE have been shown to be very accurate, ${ }^{2,3)}$ it is adequate to accomplish the guidance of ballistic reentry motion. The equations of motion for reentry problems involve, in general, a set of highly non-linear differential equations. The method of matched asymptotic expansions (MAE) has been proved to be a powerful method in analyzing the motion of reentry vehicles. However, there is a difficulty in generating solutions to go beyond the first-order solution in reentry problems. ${ }^{11)}$ Using an improved method of MAE, we are able to surpass the difficulty for higher-order solutions. ${ }^{12)}$ In this paper, we will apply the iMAE solutions to develop explicit guidance for ballistic reentry vehicles. The accuracy of ballistic trajectory and the intensity of system disturbance due to atmospheric variations are discussed as well.

\section{Equations of Motion for Ballistic Entry}

To develop explicit guidance for ballistic entry, we first consider the general case of a planar entry trajectory into a non-rotating planetary atmosphere. Using the standard notation as shown in Fig. 2, the following governing equations are given as:

$$
\begin{aligned}
\frac{\mathrm{d} r}{\mathrm{~d} t} & =V \sin \gamma \\
\frac{\mathrm{d} \theta}{\mathrm{d} t} & =\frac{V \cos \gamma}{r} \\
\frac{\mathrm{d} V}{\mathrm{~d} t} & =-\frac{\rho A C_{\mathrm{D}} V^{2}}{2 m}-g \sin \gamma \\
V \frac{\mathrm{d} \gamma}{\mathrm{d} t} & =\frac{\rho A C_{\mathrm{L}} V^{2}}{2 m}-\left(g-\frac{V^{2}}{r}\right) \cos \gamma
\end{aligned}
$$

If we define the dimensionless variables $u=V^{2} / g_{\mathrm{s}} r_{\mathrm{s}}$, and $h=\left(r-r_{\mathrm{s}}\right) / r_{\mathrm{s}}$ for the speed and the altitude with subscript $\mathrm{s}$ for reference at sea level, the governing equations for ballistic entry can be written in the dimensionless form: 


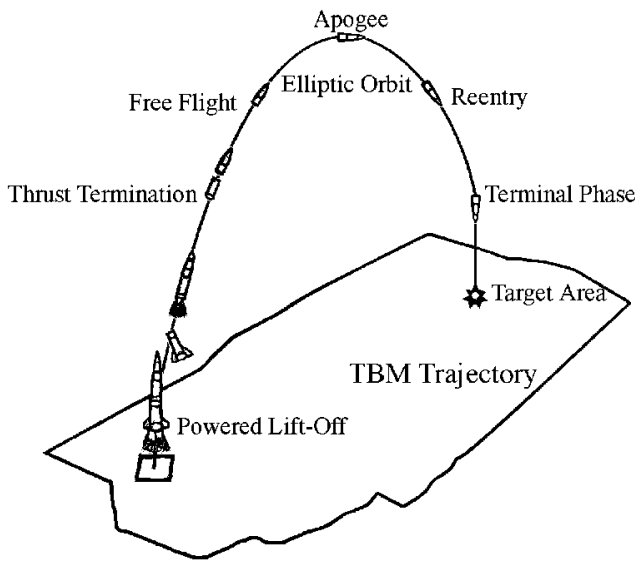

Fig. 1. The trajectory of tactical ballistic missiles.

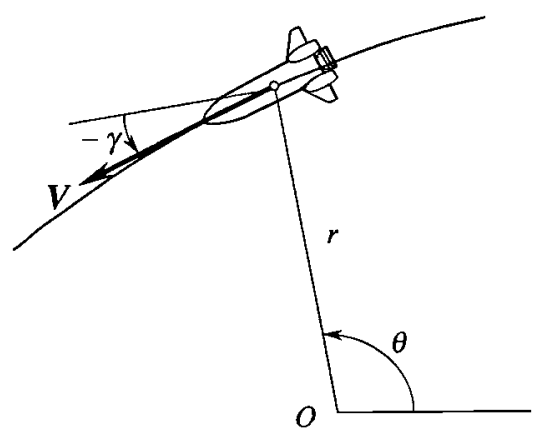

Fig. 2. Trajectory variables.

$$
\begin{aligned}
\frac{\mathrm{d} \theta}{\mathrm{d} h} & =\frac{1}{(1+h) \tan \gamma} \\
\frac{\mathrm{d} u}{\mathrm{~d} h} & =-\frac{2}{(1+h)^{2}}-\frac{\delta B_{\mathrm{m}} u e^{-h / \varepsilon}}{\varepsilon \sin \gamma} \\
\frac{\mathrm{d} \gamma}{\mathrm{d} h} & =\left\{\frac{1}{(1+h)}-\frac{1}{u(1+h)^{2}}\right\} \frac{1}{\tan \gamma}
\end{aligned}
$$

It shall be noted that we use a strictly exponential atmospheric model, $\rho=\rho_{\mathrm{s}} e^{-h / \varepsilon}$, where $\varepsilon=1 / \beta r_{\mathrm{s}}$, and $\varepsilon$ is a small dimensionless parameter. For the Earth's atmosphere, we have the practical value of $\beta r_{\mathrm{s}}=900$. The Newtonian inverse-squared gravitational field is used in the equations as given in Vinh and Kuo, 1997; ${ }^{2)}$ and Kuo and Yang, 2000. ${ }^{3)}$ To characterize the ballistic projectile, we used a constant dimensionless coefficient as the physical parameter of the vehicle, which is defined as $B_{\mathrm{m}}=\rho_{\mathrm{s}} A C_{\mathrm{D}_{\text {ref }}} / m \beta . \delta$ is the drag control defined by:

$$
\delta=\frac{C_{\mathrm{D}}}{C_{\mathrm{D}_{\mathrm{ref}}}}
$$

and subject to the constraint

$$
\frac{C_{\mathrm{D}_{0}}}{C_{\mathrm{D}_{\mathrm{ref}}}} \leq \delta \leq \frac{C_{\mathrm{D}_{\max }}}{C_{\mathrm{D}_{\mathrm{ref}}}}
$$

The system of Eqs. (2)-(4) constitutes the most appropriate dimensionless system of equations for analyzing ballistic entry with drag modulation.

\section{First-order Composite Solutions}

The iMAE solutions have been obtained by the authors, and they have been presented in a previous paper. ${ }^{3)}$ Here, the procedures of deriving the iMAE solutions will be introduced briefly.

\subsection{Outer expansion (Keplerian region)}

The outer expansion describes the behavior of motion in the region near the vacuum. The aerodynamic force is negligible compared to the combined gravitational and centrifugal forces at high altitude. Thus, let $e^{-h / \varepsilon} \rightarrow 0$ in Eq. (2). Therefore, the outer expansion is obtained by repeated application of the outer limit, which is defined as the limit when $\varepsilon \rightarrow 0$ with the variable $h$ and other dimensionless quantities are held fixed. Assume the following expansion:

$$
x=x_{\mathrm{o}}(h)+\varepsilon x_{1}(h)+\varepsilon^{2} x_{2}(h)+\ldots
$$

By substituting the expansion for $u$ and $\gamma$ in Eq. (2) and taking the outer limit, the first-order equations of the outer expansion are:

$$
\begin{aligned}
& \frac{\mathrm{d} u_{\mathrm{o}}}{\mathrm{d} h}=-\frac{2}{(1+h)^{2}} \\
& \frac{\mathrm{d} \gamma_{\mathrm{o}}}{\mathrm{d} h}=\left\{\frac{1}{(1+h)}-\frac{1}{u_{\mathrm{o}}(1+h)^{2}}\right\} \frac{1}{\tan \gamma_{\mathrm{o}}}
\end{aligned}
$$

These are the equations of Keplerian motion with the outer solutions:

$$
u_{\mathrm{o}}=\frac{2}{(1+h)}+C_{1}, \cos \gamma_{\mathrm{o}}=\frac{C_{2}}{\sqrt{C_{1}(1+h)^{2}+2(1+h)}}
$$

where, $C_{1}$ and $C_{2}$ are integration constants.

\subsection{Inner expansion (aerodynamic predominant re- gion)}

The aerodynamic force is stronger than the gravitational force near the surface of the planet. The inner expansion is obtained by repeated application of the inner limit, which is defined as the limit when $\varepsilon \rightarrow 0$ with the new altitude variable $\bar{h}=h / \varepsilon$, and the other dimensionless variables are held fixed. We assume the following expansion:

$$
x=\bar{x}_{\mathrm{o}}(\bar{h})+\varepsilon \bar{x}_{1}(\bar{h})+\varepsilon^{2} \bar{x}_{2}(\bar{h})+\ldots
$$

By substituting the expansion for $u$ and $\gamma$ in Eq. (2) and taking the inner limit, the first-order equations of the inner expansion are:

$$
\frac{\mathrm{d} \bar{u}_{\mathrm{o}}}{\mathrm{d} \bar{h}}=-\frac{\delta B_{\mathrm{m}} \bar{u}_{\mathrm{o}} e^{-\bar{h}}}{\sin \bar{\gamma}_{\mathrm{o}}}, \text { and } \frac{\mathrm{d} \bar{\gamma}_{\mathrm{o}}}{\mathrm{d} \bar{h}}=0
$$

Thus, the inner solutions can be obtained as:

$$
\bar{u}_{\mathrm{o}}=\bar{C}_{1} e^{-x}, \text { and } \bar{\gamma}_{\mathrm{o}}=\bar{C}_{2},
$$

where, $\bar{C}_{1}$ and $\bar{C}_{2}$ are the corresponding integration constants. For simplicity of notation, we define:

$$
x=-\frac{\delta B_{\mathrm{m}} e^{-h / \varepsilon}}{\sin \bar{\gamma}_{\mathrm{o}}}
$$




\subsection{Construction of composite solutions}

In order to make the solutions uniformly valid over both the outer and inner regions, we construct the composite solutions by totaling the outer and inner solutions and subtracting the parts they have in common. The basic matching principle for the first-order solutions is given as follows:

$$
x_{\mathrm{c}}=x_{\mathrm{o}}+\bar{x}_{\mathrm{o}}-x_{\mathrm{oo}}
$$

where, $x_{\mathrm{oo}}$ is the common limit:

$$
x_{\mathrm{oo}}=x_{\mathrm{o}}(h \rightarrow 0)=\bar{x}_{\mathrm{o}}(\bar{h} \rightarrow \infty)
$$

Thus, upon using the matching principle, Eq. (12), we obtain the first-order composite solutions:

$$
\begin{aligned}
u_{\mathrm{c}} & =\frac{2}{1+h}+\bar{C}_{1} e^{-x}-2, \\
\cos \gamma_{\mathrm{c}} & =\frac{C_{2}}{\sqrt{C_{1}(1+h)^{2}+2(1+h)}}
\end{aligned}
$$

By applying the matching condition, Eq. (13), the relations between $C_{1}, C_{2}$ and $\bar{C}_{1}, \bar{C}_{2}$ become:

$$
2+C_{1}=\bar{C}_{1}, \text { and } \frac{C_{2}}{\sqrt{C_{1}+2}}=\cos \bar{C}_{2}
$$

\section{Second-Order Solutions}

In the classical method of MAE, the higher-order composite solutions are obtained by expanding the outer and inner expansions to a higher order in $\varepsilon$. In contrast, in the improved MAE method, we construct the second-order solutions by considering the small discrepancies between the first-order composite solutions and the exact solutions. Let

$$
\begin{aligned}
u & =u_{\mathrm{c}}+z=u_{\mathrm{o}}+\bar{u}_{\mathrm{o}}-u_{\mathrm{oo}}+z \\
\ln (\cos \gamma) & =\ln \left(\cos \gamma_{\mathrm{c}}\right)+q=\ln \left(\cos \gamma_{\mathrm{o}}\right)+q
\end{aligned}
$$

Substituting Eq. (16) for $u$ and $\gamma$ in the basic system, Eq. (2), and using the equations for outer and inner expansions for simplification, the equations of the small perturbations $z$ and $q$ become:

$$
\begin{aligned}
\frac{\mathrm{d} z}{\mathrm{~d} h} & =-\frac{\delta B_{\mathrm{m}} z e^{-h / \varepsilon}}{\varepsilon \sin \gamma}+\frac{2 h}{(1+h)} \frac{\delta B_{\mathrm{m}} e^{-h / \varepsilon}}{\varepsilon \sin \bar{\gamma}_{\mathrm{o}}} \\
\frac{d q}{d h} & =-\frac{1}{u_{0}(1+h)^{2}}+\frac{1}{u(1+h)^{2}}
\end{aligned}
$$

The initial conditions for $z$ and $q$ are $z\left(h_{i}\right)=0$, and $q\left(h_{i}\right)=0$, where the subscript $i$ denotes the entry condition. Again, we integrate the equations for the perturbations separately first in the outer region and then in the inner region. Rewriting the first equation of the perturbations, Eq. (17), in terms of $x$, yields:

$$
\frac{\mathrm{d} z}{\mathrm{~d} x}+z=\frac{2 h}{1+h}
$$

The solution for the perturbation $z$ can be integrated as:

$$
\begin{aligned}
z= & e^{-x} \int \frac{2 h}{1+h} e^{x} \mathrm{~d} x \\
= & 2 h\left(1-h+h^{2}-+\cdots\right) \\
& +2 \varepsilon\left(1-2 h+3 h^{2}-+\cdots\right) E_{i}(x) e^{-x} \\
& +C_{3} e^{-x}+O\left(\varepsilon^{2}\right) \\
= & \frac{2 h}{1+h}+\frac{2 \varepsilon}{(1+h)^{2}} E_{i}(x) e^{-x}+C_{3} e^{-x}+O\left(\varepsilon^{2}\right),
\end{aligned}
$$

where, $C_{3}$ is the constant of integration. The exponentialintegral function is defined as:

$$
E_{i}(x)=\int_{-\infty}^{x} \frac{e^{x}}{x} \mathrm{~d} x
$$

and is a tabulated function. It turns out that the improved composite solution for $u$ is such that by combining the constants $\bar{C}_{1}$ and $C_{3}$ into a new constant $\mathrm{C}_{3}$, we obtain:

$$
u=\left[C_{3}+\frac{2 \varepsilon}{(1+h)^{2}} E_{i}(x)\right] e^{-x}+O\left(\varepsilon^{2}\right)
$$

To improve the composite solution $q$ for the flight path angle, we now integrate the second equation of Eq. (17) for $q$ :

$$
\begin{aligned}
q= & \frac{1}{2} \ln u_{0}+\int \frac{\mathrm{d} h}{u(1+h)^{2}}=\frac{1}{2} \ln u_{0} \\
& +\int \frac{e^{x} \mathrm{~d} h}{C_{3}(1+h)^{2}+2 \varepsilon E_{i}(x)} \\
= & \frac{1}{2} \ln u_{0}-\frac{1}{2} \ln \left[1+\frac{2 \varepsilon}{C_{3}} E_{i}(x)\right]+\ln C_{4}
\end{aligned}
$$

Substituting the function $u(h)$ by its solution, Eq. (21), to perform the quadrature with a very minor simplification, $1+h \approx 1$, in the integration, after some mathematical operations yields the final solution:

$$
\cos \gamma=e^{\ln \cos \gamma_{\mathrm{c}}+q}=\frac{C_{4} \sqrt{u_{0}} \cos \gamma_{\mathrm{c}}}{\sqrt{1+\frac{2 \varepsilon}{C_{3}} E_{\mathrm{i}}(x)}}
$$

Using the solutions for $u_{0}$ and $\gamma_{\mathrm{c}}$, with a new constant $C_{4}$ we actually have:

$$
\cos \gamma=\frac{C_{4}}{(1+h) \sqrt{1+\frac{2 \varepsilon}{C_{3}} E_{\mathrm{i}}(x)}}
$$

Equations (21) and (24) constitute the second-order solutions for ballistic entry. The constants $C_{3}$ and $C_{4}$ are evaluated by satisfying the initial conditions of $u_{\mathrm{i}}$ and $\gamma_{\mathrm{i}}$ at $h=h_{\mathrm{i}}$.

\section{Explicit Guidance for Drag Modulation}

The explicit guidance for drag modulation of ballistic entry is considered in this section. Since the analytical solutions of ballistic entry derived by iMAE have been shown to be very accurate, it is desirable to generate autonomous guidance and control strategies under the consideration of unknown disturbances and parameter uncertainties such as atmospheric density fluctuation, aerodynamic coef- 
ficient dispersion, system modeling uncertainty, and navigation errors.

\subsection{Trajectory tracking}

In order to develop explicit guidance and control laws, the equations of motion are written by using dimensionless variables and the assumptions described in Eqs. (1)-(4):

$$
\begin{aligned}
\frac{\mathrm{d} h}{\mathrm{~d} \bar{t}} & =\sqrt{u} \sin \gamma \\
\frac{\mathrm{d} \theta}{\mathrm{d} \bar{t}} & =\frac{\sqrt{u}}{(1+h)} \cos \gamma \\
\frac{\mathrm{d} u}{\mathrm{~d} \bar{t}} & =-\frac{\delta B_{\mathrm{m}} u^{3 / 2} e^{-h / \varepsilon}}{\varepsilon}-\frac{2 \sqrt{u}}{(1+h)^{2}} \sin \gamma \\
\frac{\mathrm{d} \gamma}{\mathrm{d} \bar{t}} & =\left[\frac{\sqrt{u}}{(1+h)}-\frac{1}{\sqrt{u}(1+h)^{2}}\right] \cos \gamma,
\end{aligned}
$$

where, we have defined the dimensionless time $\bar{t} \equiv V_{\mathrm{s}} t / r_{\mathrm{s}}$, and $V_{\mathrm{s}}=\sqrt{g_{\mathrm{s}} r_{\mathrm{s}}}$ is the circular speed at the reference altitude. Now, Eq. (25) renders a suitable form to develop guidance and control laws using iMAE solutions. The control for trajectory tracking is derived through an equilibrium flight algorithm following a reference trajectory. The control is based on the radial component of the equations of motion. From Fig. 2 and Eq. (1), the radial component of the equation of motion is:

$$
m \frac{\mathrm{d}^{2} r}{\mathrm{~d} t^{2}}=-D_{r}+m \frac{(V \cos \gamma)^{2}}{r}-m g,
$$

where, $D_{\mathrm{r}}$ is the radial component of the drag. By using dimensionless variables $u$ and $h$ to replace $V$ and $r$, we obtain:

$$
\frac{\mathrm{d}^{2} h}{\mathrm{~d} \bar{t}^{2}}=-\frac{\delta B_{\mathrm{m}} u e^{-h / \varepsilon}}{\varepsilon} \sin \gamma+\frac{u \cos ^{2} \gamma}{(1+h)}-\frac{1}{(1+h)^{2}}
$$

This equation can also be derived from Eq. (25) by applying the chain rule of calculus. Define a pseudo-control as the ratio of the radial drag to the gravity force:

$$
\eta \equiv-D_{\mathrm{r}} / m g_{\mathrm{s}}=-\frac{\rho A V^{2}}{2 m g_{\mathrm{s}}} C_{\mathrm{D}} \sin \gamma
$$

For equilibrium altitude change rate, we set Eq. (27) to be zero, which gives:

$$
\eta=\frac{1}{(1+h)^{2}}-\frac{u \cos ^{2} \gamma}{(1+h)}
$$

This control will place the flight of the reentry vehicle in an equilibrium altitude change rate. In order to achieve an equilibrium altitude rate for asymptotic tracking, an altitude damper is required. ${ }^{10,13)}$ The control equation for the commanded pseudo-control is defined as:

$$
\eta_{\mathrm{c}}=\frac{1}{(1+h)^{2}}-\frac{u \cos ^{2} \gamma}{(1+h)}-K_{\mathrm{h}}\left(\dot{h}-\dot{h}_{\mathrm{ref}}\right),
$$

where, $K_{\mathrm{h}}$ is the feedback gain for altitude rate, and $\dot{h}_{\text {ref }}$ is the reference altitude rate obtained using iMAE solutions:

$$
\dot{h}_{\mathrm{ref}}=\sqrt{u_{\mathrm{c}}} \sin \gamma_{\mathrm{c}}
$$

This indicates that the reference altitude change rate is an explicit function of altitude. Substituting Eq. (30) into Eq. (26), the radial equation of motion becomes:

$$
\ddot{h}+K_{\mathrm{h}} \dot{h}=K_{\mathrm{h}} \dot{h}_{\mathrm{ref}}
$$

Equation (32) results in a linear first-order differential equation for the altitude rate tracking with a time constant chosen to achieve the desired vehicle response:

$$
\tau=1 / K_{\mathrm{h}}
$$

After using dimensionless variables, the pseudo-control in Eq. (28) is written as:

$$
\eta_{\mathrm{c}}=-\frac{\delta_{\mathrm{c}} B_{\mathrm{m}} u e^{-h / \varepsilon}}{\varepsilon} \sin \gamma
$$

This is actually the radial force required per unit weight to attain an equilibrium altitude rate. Then, the actual drag control for trajectory tracking is solved in terms of the pseudo-control:

$$
\delta_{\mathrm{c}}=-\frac{\varepsilon \csc \gamma}{B_{\mathrm{m}} u e^{-h / \varepsilon}} \eta_{\mathrm{c}}
$$

This guidance is explicit with the control law Eq. (35), since it is expressed in terms of current state instead of the derivative of the current state with respect to a nominal state. It should be mentioned that the control law has the advantage to explicitly reveal the various effects of speed, flight-path angle, local density, and parameter $B_{\mathrm{m}}$.

\section{Numerical Example}

The objective of this study is to develop an explicit guidance method and obtain feedback controls using iMAE solutions for ballistic entry against parameter uncertainties and unknown disturbances such as system modeling errors, atmospheric density fluctuation, aerodynamic coefficient dispersion, and navigation errors. As a numerical example, the case of entry from outer space to the surface of the earth is considered. The exponential density model and the 1976 U.S. Standard Atmosphere (US-76) ${ }^{14)}$ are considered for comparison. The vehicle has a maximum drag ratio of $\delta=2$ and a minimum of 0.5 , and the nominal value of the drag control $\delta_{\mathrm{c}}$ is unity for entry maneuvers to generate the reference trajectories. The assumption of drag modulation can be easily achieved using base bleeding technology, since the base pressure of a ballistic projectile can be changed over a wide range. ${ }^{15,16)}$ A typical value of the physical parameter selected for the vehicle is $B_{\mathrm{m}}=12.76$. This would provide a sensible aerodynamic force to start entry at the altitude of $120 \mathrm{~km}$. The variations of the velocity and flight-path angle as functions of altitude for various entry angles, $-\gamma_{i}=45,60,70 \mathrm{deg}$, are plotted in Figs. 3 and 4 , respectively. In both of these figures, the solid lines denote the reference trajectories generated by the iMAE method. The dashed lines denote the trajectories computed by numerical integration with feedback control under the influence of US-76 atmospheric density dispersion, while the dashed-dotted lines represent the trajectories without 

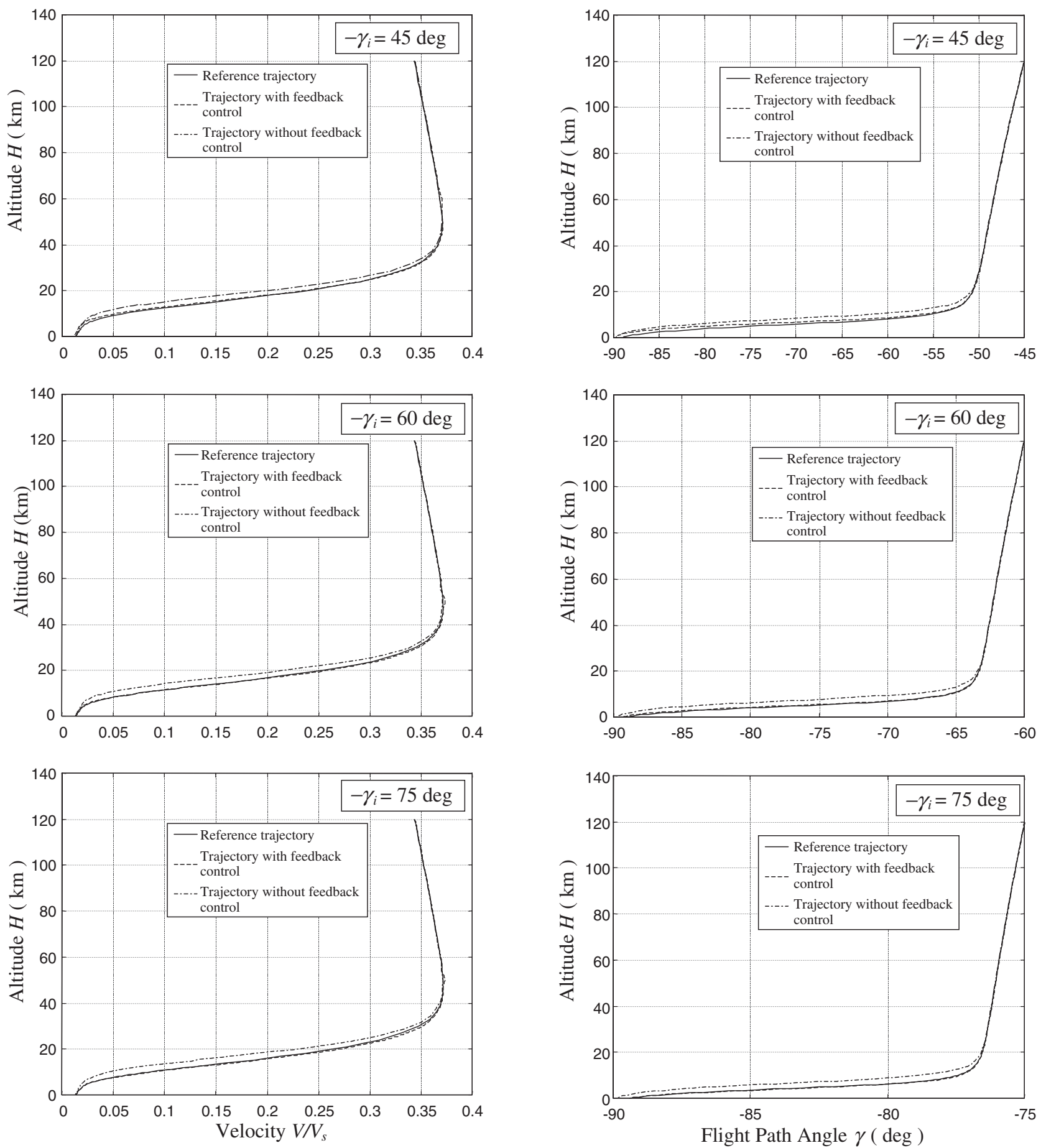

Fig. 3. Variations of velocity as functions of altitude for various entry angles.

feedback control. The feedback gain of altitude rate damper is $K_{\mathrm{h}}=5$, or equivalently, $\tau=0.2 \mathrm{~s}$ for trajectory tracking during entry. It is seen that the errors of the dashed-dotted lines propagate along the trajectories. On the other hand, a high degree of accuracy is achieved for entry with feedback control.

Figures 5 and 6 show the time histories of the state variables and the drag controls, respectively. The solid lines in the subplot of drag control in Fig. 6 are computed using the control law, Eq. (35). It is to be noted that the fluctuations within the times from 20 to $40 \mathrm{sec}$ are saturation of

Fig. 4. Variations of flight-path angle as functions of altitude for various entry angles.

control due to rare air density in a higher atmosphere. It can be seen from Eq. (35) that the exponential function of air density is positioned as the denominator of feedback control. The data in Table 1 reveal the numerical accuracy of the trajectory elements with and without feedback control under the fluctuation of US-76 atmospheric density. In each row, the upper data are absolute values for the trajectory elements, while the lower numerals are relative error with respect to the reference trajectory (Ref. traj.). From this table, we see that for the case of an initial entry angle of $60 \mathrm{deg}\left(-\gamma_{\mathrm{i}}=60 \mathrm{deg}\right)$, the relative errors of speed and 

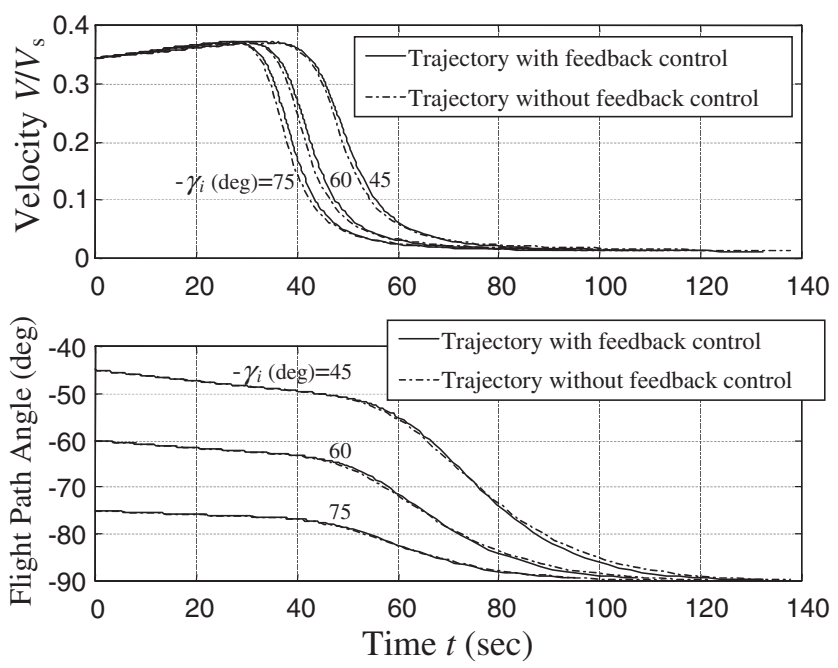

Fig. 5. Time history of the state variables. flight-path angle are merely $1.96 \%$ and $0.02 \%$, respectively, at the indexed altitude $10 \mathrm{~km}$ for the trajectory with feedback control (Traj. w/fb). In contrast to the trajectory without feedback (denoted as US-76), the relative errors are $45.54 \%$ and $5.2 \%$ for speed and flight-path angle, respectively. For the worst case, an initial entry angle of $45 \mathrm{deg}\left(-\gamma_{\mathrm{i}}=45 \mathrm{deg}\right)$, the relative errors are somewhat higher than those of entry at 60 and $75 \mathrm{deg}$. The comparison of miss distance for targeting error is listed in the last row of Table 1. For an initial entry angle of $45 \mathrm{deg}\left(-\gamma_{\mathrm{i}}=\right.$ $45 \mathrm{deg}$ ), there is not much improvement. But as the initial entry angle increases, the miss distance decreases drastically; becoming $16.8 \mathrm{~m}$ to $542 \mathrm{~m}$ for the case of entry at $75 \mathrm{deg}$. This is because the trans-atmospheric path for the case of an entry angle of $45 \mathrm{deg}$ is much longer than those of 60 and $75 \mathrm{deg}$. As expected, significant improvement in the targeting error is achieved using explicit guidance based on the iMAE solutions under the influence of density variations.

Table 1. Numerical comparison of trajectory elements for various entry angles.

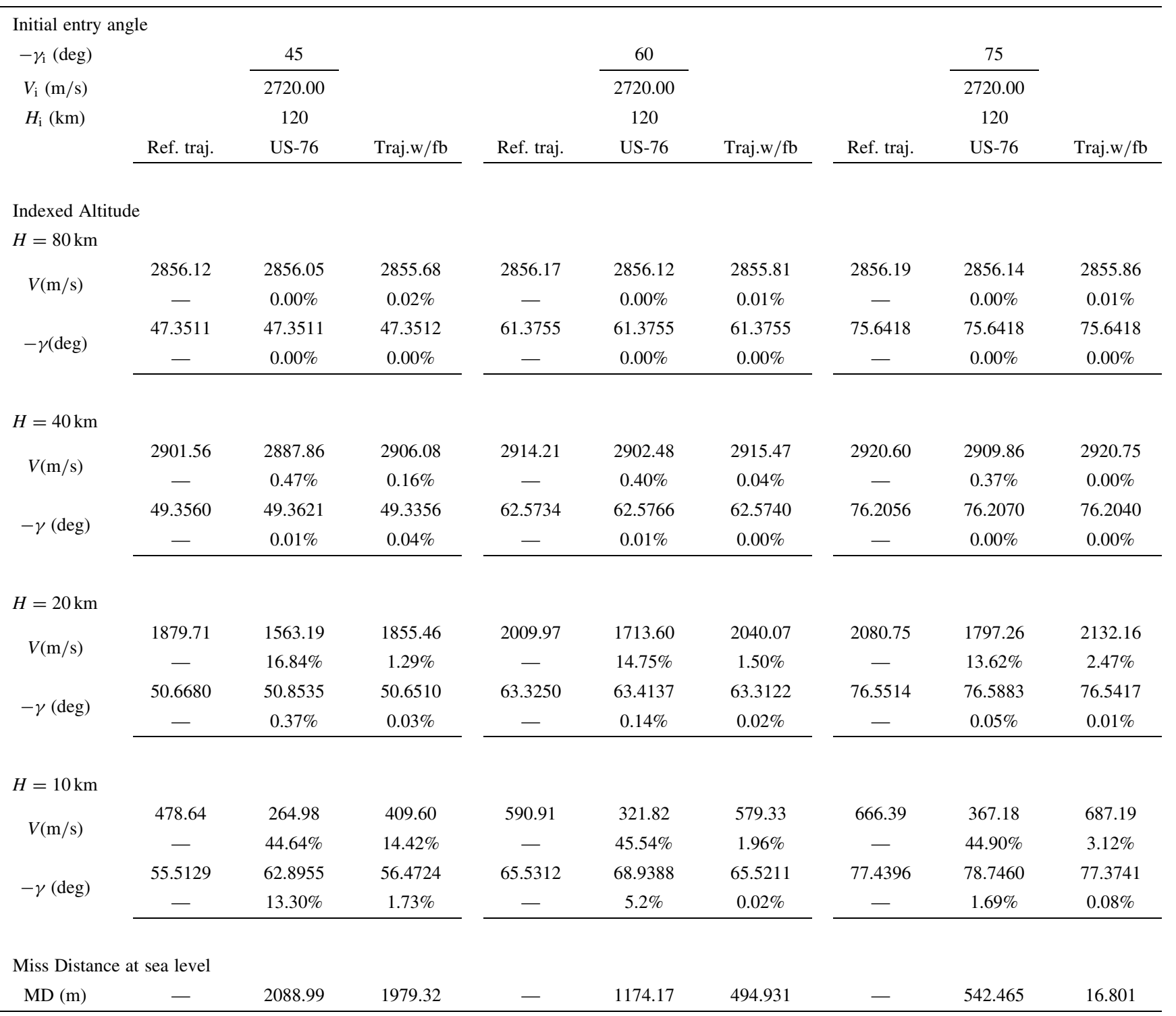



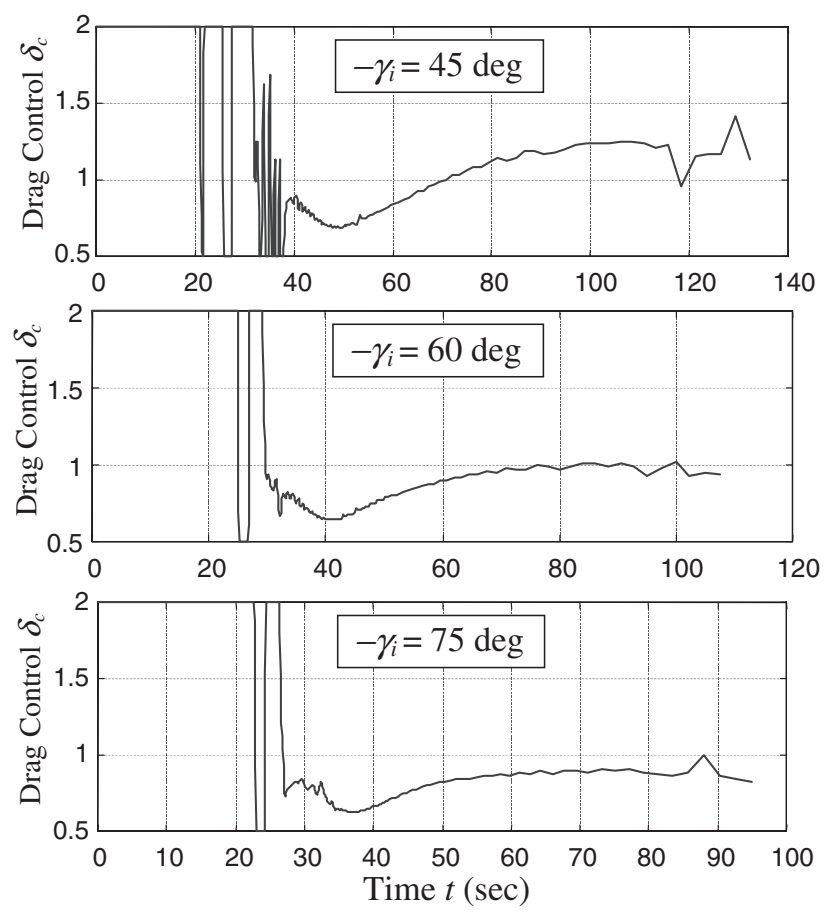

Fig. 6. Time history of the drag control.

\section{Conclusions}

An explicit guidance method for ballistic entry using iMAE solutions is developed. The guidance algorithms were tested under a wide range of targeting dispersions and atmospheric and aerodynamic variations. The results of explicit guidance using iMAE indicate that the control algorithms can effectively control the trajectory in the lower atmosphere. Highly accurate analytic formulas were derived for the case of ballistic entry and were shown to be in excellent agreement with the numerical results. A rich background of literature has developed the optimal, sub-optimal, and near-optimal guidance. It is still an open question whether or not these controls are feasible. Nevertheless, ballistic entry has an intuitive appeal. The technique presented in this paper and its analytic formulas are undoubtedly useful and amenable to mission planning and on-board guidance.

\section{Acknowledgments}

This research was supported by the National Science Council,
Taiwan, R.O.C., under Contract No. NSC-90-2212-E-014-019. The authors wish to thank the Council for their continuous encouragement and support. The authors also would like to thank the anonymous reviewers for their very helpful comments.

\section{References}

1) Zarchan, P.: Tactical and Strategic Missile Guidance, 2nd ed., AIAA Progress Series, Vol. 157, AIAA, Washington, D.C., 1994.

2) Vinh, N. X. and Kuo, Zeal-Sain: Improved Matched Asymptotic Solutions for Deceleration Control during Atmospheric Entry, Acta Astronautica, 40, 1 (1997), pp. 1-11.

3) Kuo, Zeal-Sain and Yang, Yuan-Lung: Hypervelocity Trajectories of Ballistic Entry Using Improved Matched Asymptotic Expansions, Trans. Jpn. Soc. Aeronaut. Space Sci., 42 (2000), pp. 17-24.

4) Yann-Huei Song: China's Missile Tests in the Taiwan Strait, Marine Policy, 23, 1 (1999), pp. 81-108.

5) Dougherty, J. J. and Speyer, J. L.: Near-Optimal Guidance Law for Ballistic Missile Interception, J. Guid. Control Dynam., 20, 2 (1997), pp. 355-361.

6) Kang Jianbin, Zhao Jianting and Wang, M.: An Anti-Interference Guidance Scheme for Ballistic Missile, AIAA Paper 2005-3268, 13th AIAA/CIRA International Space Planes and Hypersonic Systems and Technologies Conference, Capua, Italy, May, 2005, pp. 620-629.

7) Mundt, C., Griebel, H. and Welch, C.: Studies of Atmospheric Entry of Vehicles with Very Low Ballistic Coefficient, AIAA Paper 2005-3384, 13th AIAA/CIRA International Space Planes and Hypersonic Systems and Technologies Conference, Capua, Italy, May, 2005, pp. 1660-1671.

8) Forte, I. and Shiner, J.: Can a Mixed Guidance Strategy Improve Missile Performance, J. Guid. Control Dynam., 11 (1988), pp. 53-59.

9) Fields, J. W., Shallberg, K. W. and Ward, P. W.: Use of GPS for the Guidance of Ballistic Missiles, Proc. of the Institute of Navigation, ION GPS-96, Part 2, Vol. 2, 1996, pp. 1587-1595.

10) Kuo, Zeal-Sain and Liu, Kuo-Chung: Explicit Guidance of Aeroassisted Orbital Transfer Using Matched Asymptotic Expansions, J. Guid. Control Dynam., 25 (2002), pp. 80-87.

11) Shi, Y. Y., Pottsepp, L. and Eckstein, M. C.: A Matched Asymptotic Solution for Skipping Entry into Planetary Atmosphere, AIAA J., 9 (1971), pp. 736-738.

12) Kuo, Z.-S. and Vinh, N. X.: Improved Matched Asymptotic Solutions for Three-Dimensional Atmospheric Skip Trajectories, J. Spacecraft Rockets, 34 (1997), pp. 496-502.

13) Gamble, J. D., Cerimele, C. J., Moore, T. E. and Higgins, J.: Atmospheric Guidance Concepts for an Aeroassisted Flight Experiment, J. Astronaut. Sci., 36 (1988), pp. 45-71.

14) U.S. Standard Atmosphere, NOAA-S/T 76-1562, U.S. Government Printing Office, Washington, D.C., 1976.

15) Wei, Chin-Yi and Chang, Jeng-Ren: Wake and Base-Bleed Flow Downstream of Bluff Bodies with Different Geometry, Exp. Therm. Fluid Sci., 26 (2002), pp. 39-52.

16) Sahu, Jubaraj and Heavey, Karen R.: Numerical Investigation of Supersonic Base Flow with Base Bleed, J. Spacecraft Rockets, 34 (1997), pp. 62-69. 